\title{
Graphicality conditions for general scale-free complex networks and their application to visibility graphs
}

\author{
Miguel A. Rodríguez \\ Instituto de Física de Cantabria (IFCA), CSIC-UNICAN, E-39005 Santander, Spain
}

(Received 10 May 2016; published 26 July 2016)

\begin{abstract}
We obtain graphicality conditions for general types of scale-free networks. The same conditions obtained for uncorrelated networks are obtained in the general case. Then an upper bound relating $\gamma$, the exponent of the degree distribution, with the cutoff exponent $\kappa$, as $\kappa<1 / \gamma$ is established. This bound is valid for all networks with a well-defined power-law degree distribution in the range $\gamma \leqslant 2$. Some recent numerical research on visibility networks arising from persistent fractional Brownian motion $(\mathrm{fBm})$ processes are reviewed since they do not fulfill these conditions. As a consequence, a new relationship between the exponent $\gamma$ of the degree distribution and the Hurst exponent $H$ of the fBm process, $\gamma \lesssim 1 / H$, is postulated.
\end{abstract}

DOI: 10.1103/PhysRevE.94.012314

\section{INTRODUCTION}

The great success of complex networks in explaining properties in many diverse phenomena is because networks provide these phenomena with a rich structure able to admit a quantitative analysis. In this way, relation phenomena occurring in such distinct fields as technology, sociology, and biology [1] acquire a tangible structure amenable to analysis. Even other kinds of systems, such as time series that possess their own well-defined and long-established direct methods of analysis, are mapped to complex networks (visibility networks), with the aim of explaining phenomena not accessible to direct analysis [2,3]. Hence, the analysis and description of network structures is a subject of major interest. And the kind of network attracting most interest, since it is ubiquitously found in complex phenomena, is the so-called scale-free network. Given a network with $N$ nodes and some connections between them, defining $k_{i}$, the degree of each node $i$, as the number of edges supported by it, a scale-free network is defined as a graph whose degree distribution follows a power law $p_{k} \sim k^{-\gamma}$.

The exponent $\gamma$ is, therefore, the main characterization of a scale-free network, but other asymptotic properties related with the large-scale structure of networks are also important [4]. In particular, the asymptotic behavior of the maximum degree $k_{N}$ with the system size $N$ follows also a power law, $k_{N} \sim N^{\kappa}$, and the exponent $\kappa$ gives complementary information of the network structure. Due to the strong model dependence of $\kappa$ [5], an analysis using both exponents $\gamma$ and $\kappa$ would be a good starting point in the characterization of a network structure. Studying how these exponents behave in particular models, or when some kind of structure exists, is a methodology followed in many papers [5-16]. In general, one obtains structural bounds in the form of inequalities involving the exponents $\gamma$ and $\kappa$. These bounds are dependent on the type of network. Here we are going to consider exclusively networks with neither multiple connections nor self connections.

The main result of this paper is to complete the present incomplete picture of the role played by bounds in scale-free complex networks. It is worth remarking that, for a general type of these networks, only the so-called natural bound, $\kappa \leqslant \frac{1}{\gamma-1}$, exists [6,7], and it applies in the range $\gamma>2$. It is an effective bound in many kinds of networks, for instance, those growing with recursive methods [7,8], which are, of course, correlated, and in general for networks in the range $\gamma \geqslant 3$. This bound is not due to structural constraints but it is an inherent property of any finite degree sequence with a power-law distribution. Bounds due to structural constraints have been calculated, mainly for uncorrelated networks, using different methods: through properties of the degree-degree correlation [9-11], using statistical methods on network ensembles [12,13], or imposing graphicality conditions $[14,15]$. The structural bound for uncorrelated networks in the range $\gamma>2$ is, from Refs. [9,12], $\kappa \leqslant 1 / 2$, whereas it is $\kappa<1 / \gamma$ in the range $1<$ $\gamma \leqslant 2[11,15]$. Since natural and structural bounds arise from independent conditions, the exponent $\kappa$ of the effective bound should be taken as $\min \{1 / 2,1 /(\gamma-1)\}$ in the range $\gamma>2$. Note that for $\gamma \geqslant 3$ the effective bound is just the natural bound. To complete this picture in the range $\gamma>2$ and taking as reference the bounds of uncorrelated networks, some papers deal with the effect of correlation on these bounds. Using phenomenological arguments [9] or, more quantitatively, with explicit models $[10,16]$, it can be seen that a positive degree correlation (assortativity) produces a lower bound, whereas a negative degree correlation (disassortativity) increases the structural bound. Therefore, whereas in the range $\gamma>2$ there is a complete picture of bounds, our knowledge in the range $1<\gamma \leqslant 2$ is rather limited, only bounds for uncorrelated networks are known.

In Sec. II of this paper we extend graphicality conditions to a general case of scale free networks, establishing that $\kappa<1 / \gamma$ is a general upper bound for networks with a well-behaved power law in their degree distribution in the range $1<\gamma \leqslant 2$. As a consequence of this result, and in order to show the importance of having this class of general results, we show in Sec. III how a recent estimation of exponents in power-law distributions of visibility networks should be changed, since these conditions are not fulfilled.

\section{GRAPHICALITY CONDITIONS}

Consider a given graph with $N$ nodes whose degrees in a decreasing order are $r_{1} \geqslant r_{2} \ldots \geqslant r_{N}$. As in Ref. [15] our starting point is the graphicality conditions from the 
Erdos-Gallai (EG) theorem, which states that this sequence is graphical if it has an even sum and satisfies the EG inequalities:

$$
\sum_{i=1}^{n} r_{i} \leqslant n(n-1)+\sum_{i=n+1}^{N} \min \left[n, r_{i}\right]
$$

for any integer $n$ in the range $1 \leqslant n \leqslant N-1$. With random networks these inequalities can be analytically explored [15]. Here, dealing with the general case, we proceed in a different way. In order to connect with the standard representation of scale-free networks, we first consider the degree sequence in an increasing order $k_{1} \leqslant k_{2} \leqslant \cdots \leqslant k_{N}$, and write the EG conditions for relative degrees $q_{i}=k_{i} / k_{N}$ as

$$
\begin{gathered}
\sum_{i=n}^{N} q_{i} \leqslant k_{N}^{-1} n_{c}\left(n_{c}-1\right)+\sum_{i=1}^{n-1} q_{i} ; \quad n \leqslant m_{n}, \\
\sum_{i=n}^{N} q_{i} \leqslant k_{N}^{-1} n_{c}\left(N-m_{n}\right)+\sum_{i=1}^{m_{n}-1} q_{i} ; \quad n>m_{n},
\end{gathered}
$$

where $m_{n}$ is defined for each $n$ as the intersection of the degree with the threshold $n_{c}=N-n+1$ :

$$
\begin{gathered}
q_{m_{n}} \geqslant n_{c} / k_{N}, \\
q_{m_{n}-1}<n_{c} / k_{N} .
\end{gathered}
$$

Defining densities as $\rho(u)=\frac{1}{N} \sum_{i=1}^{N} \delta\left(u-q_{i}\right)$ and partial moments $n(s)=\int_{0}^{s} \rho(u) d u, M(s)=\int_{s}^{1} u \rho(u) d u, m(s)=$ $\int_{0}^{s} u \rho(u) d u$, we can write the graphicality condition Eqs. (1) and (2) in terms of these moments as

$$
\begin{aligned}
& N M(s) \leqslant k_{N}^{-1} n_{c}(s)\left(n_{c}(s)-1\right)+N m(s) \text { if } s \leqslant t_{s}, \\
& N M(s) \leqslant k_{N}^{-1} n_{c}(s)\left(n_{c}\left(t_{s}\right)+1\right)+N m\left(t_{s}\right) \text { if } s>t_{s},
\end{aligned}
$$

with $n_{c}(s)=N[1-n(s)]$. When $s \in\left(q_{n-1}, q_{n}\right)$ and $t_{s} \in$ $\left(q_{m_{n}-1}, q_{m_{n}}\right)$, Eqs. (3) and (4) reproduce the graphicality condition Eqs. (1) and (2). The same occurs when working with coarse-grained densities. For the sake of brevity let us consider only the first case. Consider any partition of the interval $\left[q_{1}, q_{N}\right]$ in $L$ subintervals $\left\{\left[q_{i, 1}, q_{i, l_{i}}\right]\right\}$, each one with $l_{i}$ nodes. Defining a coarse-grained density as $\rho_{\Delta}(u)=\frac{1}{N} \sum_{i=1}^{M} l_{i} \delta\left(u-\bar{q}_{i}\right)$, with $\bar{q}_{i}=\frac{1}{l_{i}} \sum_{j} q_{i, j}$ and $\Delta=\max \left\{\bar{q}_{i+1}-\bar{q}_{i}\right\}$ we can easily see that using this coarse density instead of $\rho$ graphicality inequalities are reproduced: that is, when $s \in\left(\bar{q}_{i-1}, \bar{q}_{i}\right)$, Eq. (1) is reproduced for an index $n$ corresponding to the first point of the $i$ th interval, $q_{n} \equiv q_{i, 1}$. So, in each partition only the first points of every interval $\left\{q_{i, 1}\right\}$ are reproduced. To ensure graphicality an ensemble of partitions, covering the whole set of nodes with the first node of each interval, should be used. Finally, from Eqs. (3) and (4) it is easy to see if these inequalities hold or not in the asymptotic limit $N \rightarrow \infty$. Taking the behavior of the density in a general form as $\rho(v) \sim N^{v} \rho_{0}(v), \rho_{0}(v)$ being a nonnormalized distribution with a power-law shape, $\rho_{0}(v) \sim v^{-\gamma}$, we have $n(s) \sim N^{v}, M(s) \sim N^{v}$, and $m(s) \sim$ $N^{v}$ for $\gamma \leqslant 2$ and $m(s) \sim N^{v} q_{1}^{2-\gamma}$ for $\gamma>2$. Substituting these approximations in both inequalities with $q_{1} \sim 1 / k_{N}$ and $k_{N} \sim N^{\kappa}$, we obtain the following inequalities:

$$
\begin{gathered}
1 \leqslant A N^{1-\nu-\kappa}+B \text { if } \gamma \leqslant 2, \\
1 \leqslant C N^{1-\nu-\kappa}+D N^{\kappa(\gamma-2)} \text { if } \gamma>2,
\end{gathered}
$$

where in the limit $N \rightarrow \infty, A, B, C, D$ are positive finite values dependent on the form of the distribution and $s$. The first conclusion is very clear: for $\gamma>2$ the EG conditions always hold independently of the values of $\nu$. For $\gamma \leqslant 2$ and $\nu+\kappa<1$ the EG conditions are also achieved. For $\gamma \leqslant 2$ and $v+\kappa \geqslant 1$ the EG conditions are not fulfilled, since in the asymptotic limit and for a small enough $s$, the following inequalities:

$$
\int_{s}^{1} u \rho_{0}(v) d v \leqslant\left[\int_{0}^{s} \rho_{0}(v) d v\right]^{2}+\int_{0}^{s} v \rho_{0}(v) d v
$$

for $v+\kappa=1$ and

$$
\int_{s}^{1} u \rho_{0}(v) d v \leqslant \int_{0}^{s} v \rho_{0}(v) d v
$$

for $v+\kappa>1$, cannot be fulfilled. The same reasoning is valid for the coarse-grained distribution $\rho_{\Delta}$. Since in a well-behaved power-law distribution these inequalities are not dependent on details of coarsening, EG conditions apply for any point of the original distribution.

Note that these graphicality conditions apply to a general case in which the relative degree distribution $\rho(v)$ (also coarse grained distributions) scales as $\rho(v) \sim N^{v} v^{-\gamma}$. Focusing on the most common case where the degree distribution $\rho(u)=\frac{1}{N} \sum_{i=1}^{N} \delta\left(u-k_{i}\right)$ and the maximum degree $k_{N}$ show a well-defined power law $\rho(u) \sim u^{-\gamma}, k_{N} \sim N^{\kappa}$, we obtain $v=\kappa(\gamma-1)$ and hence graphicality conditions state that for $\gamma>2$ and $\gamma \leqslant 2$ with $\kappa<1 / \gamma,\left\{k_{i}\right\}$ sequences are always graphical, whereas they are not in any other case.

\section{TIME SERIES AND VISIBILITY NETWORKS}

Visibility graphs are produced from time series by considering that two data points that are mutually visible are connected. In more concrete terms, given a time series $\left\{y_{t_{1}}, y_{t_{2}}, \ldots, y_{t_{N}}\right\}$, any pair of data points, $y_{t_{i}}, y_{t_{j}}\left(t_{i}<t_{j}\right)$, are mutually visible and therefore connected in the corresponding graph, if and only if for any intermediate $t_{k}, t_{i}<t_{k}<t_{j}$,

$$
y_{t_{k}}<y_{t_{i}}+\left(y_{t_{j}}-y_{t_{i}}\right) \frac{t_{k}-t_{i}}{t_{j}-t_{i}} .
$$

Note that by definition the visibility graph is undirected and always connected, since each node is linked at least with its neighbors. Then a visibility network always maintains some correlation, even when the corresponding time series is random. In fact, the Pearson correlation coefficient for visibility networks of fractional Brownian motion (fBm) and fractional Brownian noise ( $\mathrm{fBn}$ ) processes is nearly constant with a value close to 0.2 , so they are weakly assortative networks. Another important property is the invariance of visibility graphs under affine transformation of the series data, so local transformations in time series are irrelevant in their corresponding visibility graph. That is, visibility graphs are 


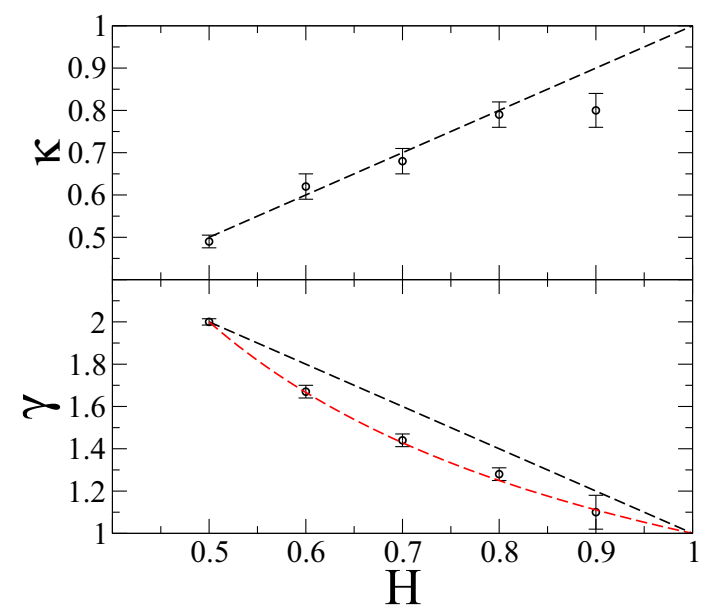

FIG. 1. Exponents $\kappa$ and $\gamma$ of visibility networks arising from persistent $\mathrm{fBm}$ processes with $1>H \geqslant 0.5$. Points are numerical results with errors; black dashed straight lines are extrapolated theoretical results, $\kappa=H, \gamma=3-2 H$; and the red dashed curve is the value postulated in this paper to fulfill graphicality conditions, $\gamma=$ $1 / H . \kappa$ values are obtained from a fitting of a log-log representation of $\left\langle k_{N}\right\rangle$ against $N$, using 100 samples and $N=2^{13}, 2^{14}, 2^{15}, 2^{16}$. $\gamma$ values are obtained as shown in Fig. 2.

only sensitive to nonaffine properties of time series. Despite this divergence, another expected property is to have a close connection between properties of time series and those of their respective visibility graphs. This connection is not so strong as to connect random time series with random visibility networks [3], but one expects that self-affine series give rise to something equivalent in networks. A key result confirming these expectations was the relationship $\gamma=3-2 H[3,17]$ between the Hurst exponent $H$ of self-affine series produced by $\mathrm{fBm}$ processes and the exponent $\gamma$ of the degree distribution of the associated visibility graph (which is a power law).

This relationship can be seen even closer if, instead of $\gamma$, one estimates the exponent $\kappa$, obtaining $\kappa=H$ (Fig. 1). From this result we can draw two interesting conclusions. First, it confirms the high dependence of the cutoff exponent on the network model [5]. Second, it gives a physical interpretation to the connection between time series and visibility networks. Note that the number of points that are visible from the point with maximum visibility, $k_{N}$, scales as $N^{\kappa}$, so the exponent $\kappa$ can be interpreted as the fractal dimension of this visible zone. Self-affinity of time series with exponent $H$ implies a maximum visibility zone with fractal dimension $H$. This result has already been used in the heuristic derivation of the linear relation $\gamma=3-2 H$ for the estimation of the number of visible nodes from a hub of the network [17].

In our numerical analysis $\mathrm{fBm}$ processes are generated with the Lebinson's algorithm implemented in Mathwork Matlab. The point $H=1$ is unstable and therefore values such as $H=$ 0.9 , close to this point, should be carefully treated. Note that in Fig. 1 errors are only due to fitting processes. The discrepancy with the estimated exponent for $H=0.9$ is due to its proximity to the unstable point. But something is not correct since with these values of exponents, $\gamma=3-2 H$ and $\kappa=H$, and in the persistent case $(0.5<H<1)$, graphicality conditions

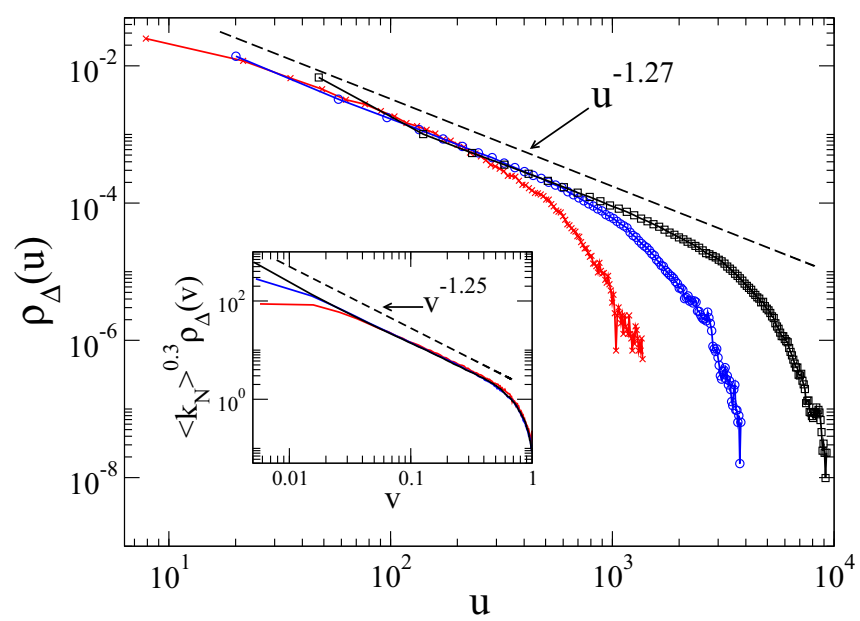

FIG. 2. Averaged coarse-grained density of degrees $\left\{k_{i}\right\}$ for a visibility network produced from $\mathrm{fBm}$ processes with $H=0.8 . N=$ $2^{12}$ (red crosses), $2^{14}$ (blue circles), and $2^{16}$ (black squares). Averaging over 100 samples and with a partition of homogeneous intervals with $\Delta=\left[\max \left(k_{N}\right)-\min \left(k_{1}\right)\right] / 100$. Inset: collapsed averaged coarse density of relative degrees $\left\{q_{i}\right\}$ with $\Delta=\left[\max \left(q_{N}\right)-\min \left(q_{1}\right)\right] / 100$. From these figures we obtain $\theta=0, \gamma_{1}=1.27$ [from $\rho_{\Delta}(u)$ ], $\gamma_{2}=$ $1.25, v=0.3, \gamma_{3}=v+1=1.3$ [from $\rho_{\Delta}(v)$ ]. Therefore, we have three estimations of $\gamma$ from which we obtain an accurate value of $\gamma_{\text {num }}$ with errors.

$\kappa<1 / \gamma$, that in principle apply in this case, are not fulfilled. A more detailed inspection of numerical results suggests the convenience of distinguishing between true numerical results, producing $\gamma_{\text {num }}$ and $\kappa_{\text {num }}$ exponents, and theoretical exponents, $\gamma_{\text {th }}$ and $\kappa_{\text {th }}$, that are an extrapolation of the numerical ones. Differences between numerical and theoretical values are attributed to statistical errors, that could be minimized using better sampling, and to finite-size effects. Certainly, finite-size effects may be the cause of this discrepancy in the case of antipersistent $\mathrm{fBm}$ processes $(0<H<1 / 2)$ but this is not

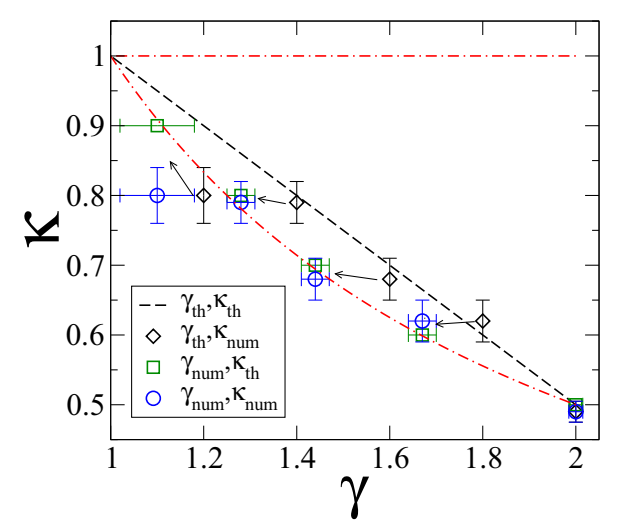

FIG. 3. $\kappa$ versus $\gamma$ plot. Red dot-dashed lines are barriers. The upper is the natural $\kappa \leqslant 1$ and the lower, $\kappa \leqslant 1 / \gamma$, is due to graphicality constraints. Points including $\gamma_{\text {th }}=3-2 H$ are above the graphicality barrier. Taking a new $\gamma_{\text {th }}^{\prime} \lesssim 1 / H$ the theoretical curve $\left\{\gamma_{\mathrm{th}}^{\prime}, \kappa_{\mathrm{th}}\right\}$ becomes just below the graphicality barrier. The extrapolation $\gamma_{\text {num }} \rightarrow \gamma_{\text {th }}^{\prime}$ seems to be the correct one. 


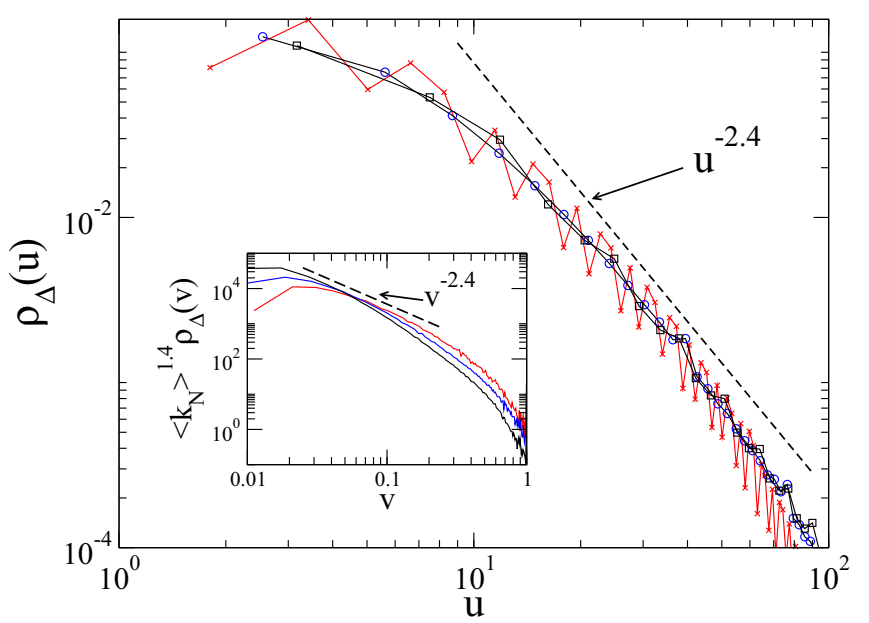

FIG. 4. As in Fig. 2, but now with $H=0.3$. In contrast to this figure a true power law is not observed here for these sizes. The possible straight line in the main figure is shorter than one decade. Only one point is collapsed in the inset and the slope of the enveloping line (2.4) is in agreement with the linear relation $\gamma=3-2 H$.

so in the persistent case. In Fig. 2 we observe that finite size effects do not change the numerical value of $\gamma_{\text {num }}$ and therefore its extrapolation to the theoretical value should be questioned. The lower frame of Fig. 1 defines clearly the numerical value $\gamma_{\text {num }} \approx 1 / H$. Concluding, Fig. 3 shows a clear picture of the problem and its solution. When the value $\gamma_{\text {th }}=3-2 H$ is used, either with $\kappa_{\text {th }}$ or $\kappa_{\text {num }}$, graphicality conditions are exceeded, whereas with $\gamma_{\text {th }}^{\prime} \lesssim 1 / H$ they are fulfilled. Note that the equality $\gamma_{\text {th }}^{\prime}=1 / H$ is not in agreement with the graphical bound, so with $\gamma_{\text {th }}^{\prime} \lesssim 1 / H$ we want to indicate a value of $\gamma_{\text {th }}^{\prime}$ very close to but lower than $1 / H$. In this way the numerical result $\gamma_{\text {num }} \approx 1 / H$ and the graphical condition $\gamma_{\text {th }}<1 / \kappa=1 / H$ are compatible.

In the case of antipersistent processes graphicality conditions do not impose any bound, so the only constraint is the natural bound $\gamma \leqslant \frac{1+H}{H}$ that is far from the expected linear result $\gamma=3-2 H$. Although this case is not relevant to our study of graphicality it is worth noting that, in contrast to the persistent case, here finite-size effects are so strong that it is even difficult to identify a true power law in the degree distribution (Fig. 4). What one sees in this range is that the exponent of the enveloping line in the collapsed distributions (inset of Fig. 4) follows the linear relation $\gamma=3-2 H$. A more detailed analysis, which will appear in a further publication, is beyond the scope of this paper.
Two facts are remarkable when analyzing these results. First, that the values of $\kappa_{\text {num }}$ and $\gamma_{\text {num }}$ are just on the edge of the graphicality barrier, that is also a structural bound for uncorrelated networks. One would expect that, as happens in $\gamma>2$, and due to the assortative character of visibility networks, these values would be well below this bound. As it is not the case, the role of correlations in this range becomes an open question. Second, concerning the robustness of exponents $\gamma$ and $\kappa$, it has been intuitively assumed that the power-law exponent $\gamma$ is more robust against changes in the models than the cutoff exponent $\kappa$. In fact, it is said that $\kappa$ is an exponent strongly dependent on the model [5]. Our results show just the opposite. Graphicality constraints change the value $\gamma_{\text {th }} \rightarrow \gamma_{\text {th }}^{\prime}$ keeping the exponent $\kappa$ unaltered.

\section{CONCLUSIONS}

An upper bound due to graphicality constraints is established for general scale-free networks in the region $1<$ $\gamma \leqslant 2$. This bound is identical to the bound encountered for uncorrelated networks. This leaves open the interesting question of whether correlations are structurally irrelevant in this range, or simply their effects on the cutoff process become collapsed by graphical conditions.

From a more practical point of view we show how the knowledge of structural bounds is useful to define exponents and properties in some limit situations as, for instance, when some extrapolation of the numerical work is necessary to arrive at a consistent theory. As a good example confirming these ideas we review an important recent result where time series and visibility networks are mutually related through their characteristic exponents. Time series are generated from fBm processes with Hurst exponent $H$. Their corresponding visibility networks are scale free with an exponent of the degree distribution given by $\gamma=3-2 H$. This revision is justified since the exponents $\gamma$ and $\kappa$ of the visibility network, in the case of persistent $\mathrm{fBm}(0<H \leqslant 1 / 2)$, do not fulfill the graphicallity conditions established before. After a more detailed revision of the numerical work a new relationship $\gamma \lesssim$ $1 / H$ instead of $\gamma=3-2 H$ is postulated. Now graphicality conditions are fulfilled.

\section{ACKNOWLEDGMENTS}

I thank J. Ramasco, J. M. López, and D. Pazó for valuable discussions. I acknowledge support from MINECO (Spain) under Projects No. Fis2014-59462-P and No. CGL201566583-R.
[1] M. E. J. Newman, Networks, An Introduction (Oxford University Press, Oxford, UK, 2013).

[2] J. Zhang and M. Small, Phys. Rev. Lett. 96, 238701 (2006).

[3] L. Lacasa, B. Luque, F. Ballesteros, J. Luque, and J. C. Nuo, Proc. Natl. Acad. Sci. USA 105, 4972 (2008).

[4] L. F. Costa, F. A. Rodrigues, G. Travieso, and P. R. Villas Boas, Adv. Phys. 56, 167 (2007).
[5] S. N. Dorogovtsev and A. V. Goltsev, Rev. Mod. Phys. 80, 1275 (2008).

[6] R. Cohen, K. Erez, D. ben-Avraham, and S. Havlin, Phys. Rev. Lett. 85, 4626 (2000).

[7] S. N. Dorogovtsev, J. F. F. Mendes, and A. N. Samukhin, Phys. Rev. E 63, 062101 (2001).

[8] B. Waclaw and I. M. Sokolov, Phys. Rev. E 75, 056114 (2007). 
[9] M. Boguňa, R. Pastor-Satorras, and A. Vespignani, Eur. Phys. J. B 38, 205 (2004).

[10] S. Weber and M. Porto, Phys. Rev. E 76, 046111 (2007).

[11] H. Seyed-allaei, G. Bianconi, and M. Marsili, Phys. Rev. E 73, 046113 (2006).

[12] Z. Burda and A. Krzywicki, Phys. Rev. E 67, 046118 (2003).

[13] S. N. Dorogovtsev, J. F. F. Mendes, A. M. Povolotsky, and A. N. Samukhin, Phys. Rev. Lett. 95, 195701 (2005).
[14] C. I. Del Genio, T. Gross, and K. E. Bassler, Phys. Rev. Lett. 107, 178701 (2011).

[15] Y. Baek, D. Kim, M. Ha, and H. Jeong, Phys. Rev. Lett. 109, 118701 (2012).

[16] J. Menche, A. Valleriani, and R. Lipowsky, Phys. Rev. E 81, 046103 (2010).

[17] L. Lacasa, B. Luque, J. Luque, and J. C. Nuno, Europhys. Lett. 86, 30001 (2009). 